\title{
Predictive Model for Dyslexia from Eye Fixation Events
}

\author{
Jothi Prabha A, Bhargavi R, Harish B
}

\begin{abstract}
Dyslexia is a specific learning disorder where the individual often find difficulty in spelling and reading words fluently. Dyslexia is non-curable but with right remedial support, dyslexics can become highly successful in academics and life. Eye movement patterns during reading process can provide an in-depth understanding about reading disorders caused by dyslexia. Eye movements can be captured using eye-tracker, from which the relationship between how eyes move with respect to the words they read can be understood. In this work, a set of binocular fixation and saccade features were extracted from raw eye tracking data based on statistical measures. Machine learning algorithms such as Random Forest Classifier (RF), Support Vector Machine (SVM) for classification and K-Nearest Neighbor (KNN) were analyzed to output classification models for prediction of dyslexia. KNN gave higher levels of accuracy of $95 \%$ compared to SVM and RF over a small feature set of features related to fixations and saccades. These eye features can be used as a basis for developing screening means for prediction of dyslexia. Prediction of dyslexia at an early stage can help children to go for remediation which helps them for academic excellence.
\end{abstract}

Keywords: Dyslexia, Eye movements, KNN, RF, SVM

\section{INTRODUCTION}

$\mathrm{E}_{\mathrm{ye}}$ movements while reading was first described by Emile Java in the year 1879.He was the first to observe that eye movements are not continuous but has different patterns like rapid movements called saccades and stop duration called as fixations. The relationship between human eye movements and their cognitive abilities were focused since 1970[1]. The advent of personal computers at low price led to extensive research on different groups of users mainly people with reading disabilities. It is also widely used by business organizations to improve their business by understanding the interest of their customers from their reading habits [2] [3] [4]. Eye movements are used for prediction of dyslexia, cognitive and Alzheimer. Analyzing eye movement patterns using machine learning algorithms can help in better prediction of dyslexia [4][5].

Dyslexia is specific learning disorder where an individual with good intelligence quotient can struggle to read fluently. Dyslexics find it difficult to match the letters on a page with

Revised Manuscript Received on December 16, 2019.

* Correspondence Author

Jothi Prabha A*, School of Computing Sciences \& Engineering, Vellore Institute of Technology, Chennai Campus, India, Email: jothiprabha@gmail.com

Bhargavi R, School of Computing Sciences \& Engineering, Vellore Institute of Technology, Chennai Campus, India, Email: bhargavi.r@vit.ac.in

Harish B, School of Computing Sciences \& Engineering, Vellore Institute of Technology, Chennai Campus, India, Email: harish.b2015@vit.ac.in its sound which causes them to struggle to read and spell words correctly. Dyslexia cannot be cured but when diagnosed earlier and given right remedial support can help them become successful [6][7][8]. Various computational models to prediction of dyslexia exist in literature but none of them are remarkable. The research done in this paper is to come up with a machine learning model for detection of dyslexia from eye movement reading patterns. This paper proposes to use statistical measures to derive the eye movements and features from the raw eye tracking data and develop a machine learning model for classification of dyslexia using these features and movements.

In the second section of this paper, relevant literature work carried out on eye tracking studies in dyslexia, medicine and other applications are discussed. The third and fourth sections of the paper discuss the data collection and methodology respectively. Fourth and fifth section shows the results of the experimentation and comparative analysis with other existing models. Sixth section discuss the conclusion of this research work

\section{RELATED WORK}

Eye movements of Chinese children both dyslexic and controls were observed while performing Stroop Color and Word test (SCWT). Eye Link II eye tracker was used to record the eye movements. It has been observed that Chinese dyslexic children show low accuracy and slow response time. Dyslexic children have abnormal eye movements such as a greater number of fixations and saccades, lower frequency fixations and short mean saccade distance. It has also been observed that dyslexic children have Visual Attention span disorder which contributes to abnormal eye movements [9][10][11].

A screening tool for Dyslexia has been built by recording the eye movements of children during reading silently. There are many features that correlate the eye movement with the words they read. A larger feature set was derived from statistical properties of fixations and saccades. Lasso regression is used for dimensionality reduction to get a lesser feature set that include median and mean of saccade length, count of short forward movements and total count of fixation words. Classification was performed using machine learning algorithm SVM [12].

Eye movements were observed while performing letter naming speed test conducted on children with and without dyslexia. Naming speed is the ability to identify or name a set of letters quickly and accurately. Analysis of eye movements was done, and it has been observed that increased similarity in visual representation of letters decreased the speed of naming letters. Children with dyslexia were observed to have more pause times while reading, 


\section{Predictive Model for Dyslexia from Eye Fixation Events}

more fixations and more regression errors when compared to the control group. Pause times and fixation duration were observed to be primary predictors for reading [13].

In this work, prediction of reading mistake has been done from real world teaching using eye gaze features. Ensemble of different classifiers was used for classification. The data was collected in a noisy environment and have few missing data. The model has shown good performance even with noise. Fifteen features related to fixation where considered as major ones for prediction. This model can be used for personalized text simplification [14].

A study has been conducted to explore the association between eye movements and learning difficulties. It has been observed that the eye movement of dyslexic individuals shall contribute for difficulties in visual reading. It has been reported that the difference in eye movement pattern for dyslexics is because of visual magnocellular impairment. Magnocellular system plays a vital role in visual guidance of eye movements. However, this was tested on a small sample and if the child has other phonological and auditory disorders, it is hard to detect magnocellular impairment [15].

This work has studied saccadic movements vertically in dyslexic kids. Infrared video-oculography system is used to capture the left and right eye movement patterns It has been reported that dyslexic children have longer latency compared to control group. From the findings it has been suggested that impairment in cortical areas could be a cause of this abnormal vertical saccades' performance in dyslexics [16].

Screening of dyslexia from their eye movements and machine learning analysis has been done for prediction of dyslexia. Horizontal and vertical eye movements are recorded and analyzed using Support vector machine (SVM) algorithm. Feature reduction is done by using SVMRecursive Feature Elimination (RFE) algorithm. Accuracy of 94\% has been achieved using linear SVM [17].

Poor binocular synchronization of saccades has been observed in dyslexic kids while performing single word reading test. Also, stereo type pattern is not observed in dyslexics as in control group. No proper divergence occurs during saccade movement and no proper convergence occurs at the end of the saccade. It has been reported that the reason for this abnormality could be due to deficits in ocular motor learning system [18].

German dyslexics were compared to English dyslexics. Eye movements of 13-year-old boys were recorded while reading text passages and list of pseudo words. It has been observed for both the cases, dyslexics were observed to have more fixations and less regression. More the length of the word and more the complexity of word caused increased number of fixations in dyslexics than normal readers. Findings show that German dyslexics have fewer regressions compared to English and Italian dyslexics and this could be due to syllabic complexity of the German language [19].

This work explores the fixation visually in dyslexics and non-dyslexics. Binocular eye movements both horizontally and vertically were recorded. Fixations and saccadic movements have been reported that dyslexics have more unwanted saccadic movements when compared to the other two groups. This poor visual fixation ability in children may be because of poor attention skills and due to immature cortical areas, which is responsible for fixation control [20].

This works explores the difficulties faced by dyslexics in interpreting graph data. Dyslexics find hard to interpret graphs when compared to normal text because of their orthogonal deficits. They find difficult to process and interpret orthographic components of graph such as axes labels and legends. Dyslexics were able to interpret graph free of orthographic components [21].

\section{DATA COLLECTION}

Ober-2, eye gaze tracker is used for capturing eye movements. The eye position is tracked for almost a minute in milliseconds $(\mathrm{t})$. The children were to read a passage that is appropriate for their age. The data used in this work was collected from the work of Benfatto, et al. [30]. The dataset contains raw recording data of 185 subjects studying grade 2 . 97 were dyslexics and 88 were non-dyslexics.

\section{METHODOLOGY}

This research work comes up with a set of features that can be used for building an accurate prediction model for dyslexia. The proposed system architecture is shown in Fig. 1. A set of fixation and saccade related features were derived from binocular eye movement data using statistical measures.

\section{A. Data Preprocessing}

The data recorded by the eye tracker has five readings Lx, Ly, Rx, Ry, t. $x$ and $y$ denote the eye coordinates on the monitor of computer. Lx is left eye's x position, Ly is left eye's y position, $\mathrm{Rx}$ is right eye's $\mathrm{x}$ position, Ry is right eye's $y$ position and $t$ is time taken for reading in seconds. When the subjects blink, the data value will be set to blank. The

\section{B. Feature Selection}

Feature selection is a process by which we can select data or attributes that contribute more for prediction or the output variable. Irrelevant and redundant features can reduce the accuracy of the predictive model. The main benefits of performing feature selection is to reduce overfitting and increases accuracy.

In this work, Recursive Feature Elimination (RFE) and cross validated selection of best features is experimented. This method removes the irrelevant or redundant features recursively. A set of features related to fixation events were derived from the raw data using statistical measures. A fixation state is when the user gazes continuously at a point for a minimum of 80 milliseconds. The fixation related parameters must be measured both horizontally and vertically for both the eyes. 


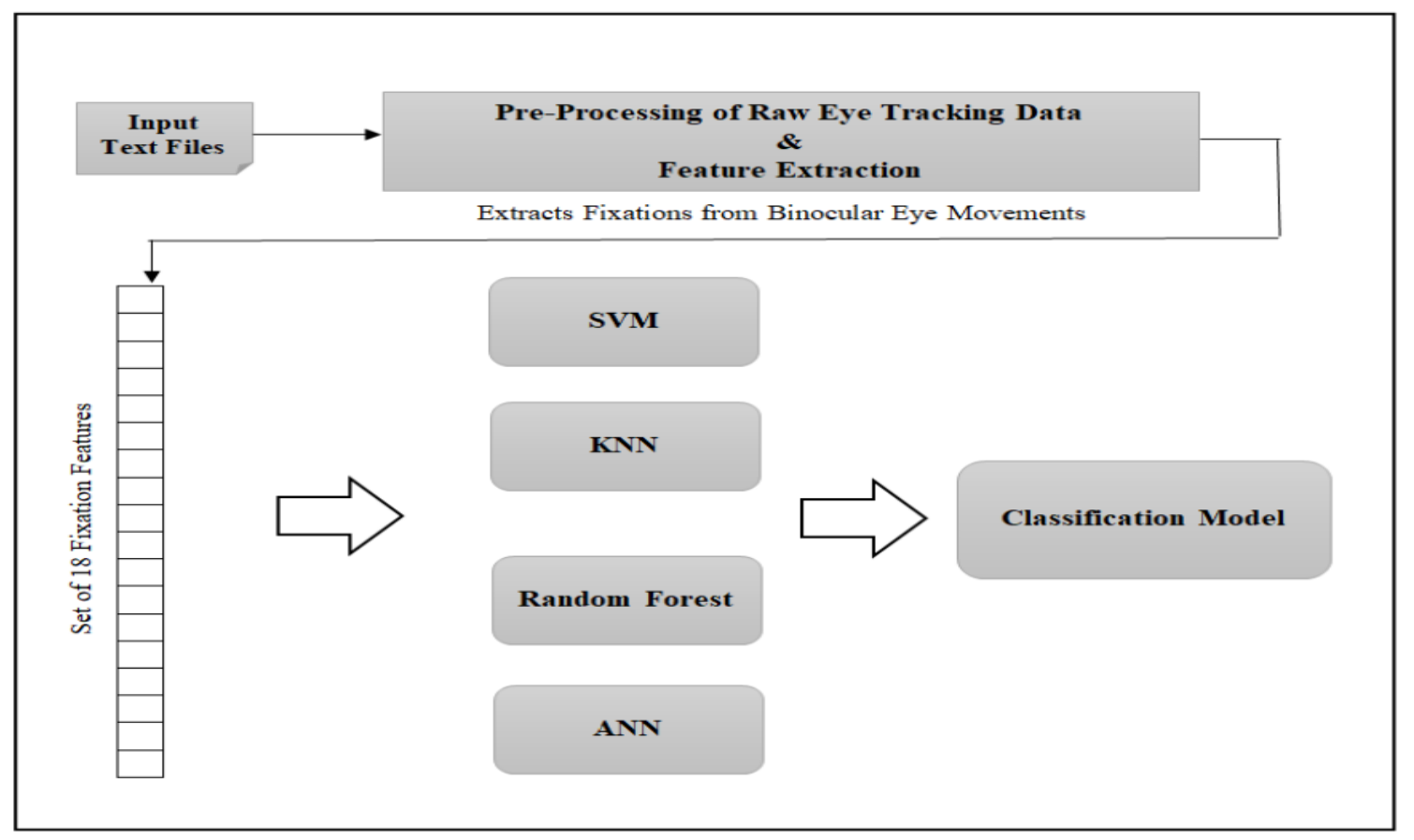

Fig. 1 Proposed system architecture

\section{Algorithm 1 Recursive Feature Elimination (RFE)}

1: for each fold do

2: Partition the dataset into training and testing records

3: Train the model to learn the training set with all features

4: Predict the testing samples

5: Determine the importance of each feature

6: for each subset size $\mathrm{Ki}, \mathrm{i}=1 . \ldots \mathrm{N}$ do

7: $\quad$ Hold the Ki most relevant features

8: $\quad$ Train the model using these Ki features

9: $\quad$ Predict the testing samples

10: end for

11: end for

12: Calculate the accuracy of testing data

13: Select the number of features to be retained

14: Predict final list of features for the model

15: Fit the model based on the optimal Ki using the training set

Fig. 2. Recursive Feature Elimination Algorithm

\section{Support Vector Machines for Classification}

SVM is a classification algorithm for both linear and non-linear data. Each piece of information is depicted as a vector of n-dimensions. For linear information, to separate all rows of a class, it draws an obviously maximum separating hyper plane. There may be several dividing lines, the one with the least classification error is the best hyperplane. By looking for maximum marginal hyper plane, SVM selectt he highest hyper plane. The margin provides the classes the biggest separation. It handles non-linear data by transforming in a greater order dimension the initial training data. For this new dimension, the linear hyper plane is being searched. For this mapping, the kernel function in SVM is helpful. Polynomial, linear, quadratic, radial basis task and Sigmoid are different kinds of kernels commonly used.
SVM has been shown to operate better with standardized information. Our information ranges from -1 to 1 and is therefore already standardized. In literature, SVM worked well with a small set of features for small datasets. SVM showed better predictive performance in disease prediction. Taking these inputs into account, SVM with different kernels such as linear, radial basis function (RBF) and sigmoid are chosen for experimentation this research work. The predictive accuracy achieved was $93.5 \%, 93 \%$ and $92 \%$ for linear, RBF and sigmoid kernels respectively.

Hybrid SVM-Kernel proposed in [30] which is a combination of linear and quadratic kernels was tested and gave an accuracy of $95 \%$.

\section{KNN for Classification}

$\mathrm{KNN}$ algorithm is widely used for both classification and regression problems. KNN algorithms classify new data points based on similarity measures such as distance function using the available data. Classification is done by majority voting technique to its neighbors. The data is assigned or labeled to the category or class which has the nearest neighbors. The main advantages of $\mathrm{KNN}$ are it is highly insensitive to outliers and does not make any assumptions about data. It works best with numeric and nominal values. In literature, KNN has been widely used in health care industry for prediction of diseases.

In this work, 5 fixation related features were given as input to KNN algorithm. The numbers of neighbors were chosen by trial and error method and the algorithm gave highest accuracy when the numbers of neighbors are 3 . The predictive accuracy achieved was $95 \%$. KNN out rated SVM and RF in terms of accuracy. 


\section{Predictive Model for Dyslexia from Eye Fixation Events}

\section{E. Random Forest for Classification}

Random forest learning model is an ensemble of decision trees that is widely used for classification problems. Random forest (RF) is a combination of bagging approach and random feature selection methods. It can identify critical variables from a big set of input variables. It is a random subspace method for constructing groups of decision trees called as decision forests. It uses bagging technique to construct every decision tree of the ensemble using sample with replacement method. The sample size is usually $64 \%$ of total instances with at least one instance in every sample. Instances in and out of a sample are called in-bag instances and out-of-bag instances respectively. Every tree serves as a base classifier for finding the class label of unknown instances. Class label is identified using majority voting technique where each classifier gives their vote for a class label. The unknown instance will be assigned to the class label with highest vote. This procedure is termed as random forest.

Random forests show improved accuracy in classification and regression problems. Main advantages of Random forest are its robustness in noisy data, and it does not cause over fitting. Over fitting refers to the model that is trained too well. It works well on training data but fails in testing data. An over fitted model show has less predictive performance due to lack of generalization. Generalization refers to the performance of the model in new data which is not part of training data. Over fitting degrades the performance of the model and adds unwanted complexity. Classification model that are over fit will tend to have less error rate in in-bag (training) instances and high error rate for the out-of-bag (testing) instances. Random forest overcomes the over fitting problem easily by averaging all the predictions to avoid biased results. Random Forest has been experimented in this work mainly to avoid over fitting as the dataset is small and Random forest have performed well in prediction of diseases.

\section{RESUlts AND Discussion}

The experimental results of the current research work are discussed in this section. In this experimental set up, dyslexics belong to positive class and non-dyslexic to negative class. Performance is evaluated by using metrics such as False Positive Rate (FPR), sensitivity, specificity, recall and False Negative Rate (FNR). The classification model was trained using Support Vector Machine, Random Forest Classifier, K-Nearest Neighbor and Artificial Neural Network. The results show that K-Nearest Neighbor Model and Hybrid SVM model used in this work achieved higher level of accuracy compared to Support Vector Machine and Random Forest. The comparative results of the experimented classification models are shown in Table I.

\section{Conclusion}

This research work identifies a set of fixation related eye features which can be used to develop a prediction model for dyslexia. Accurate prediction depends on the features which are used to build the prediction model. Hence this work focuses on identifying features that contribute for better prediction and, then build an appropriate prediction model. Fixations play a key role in prediction of dyslexia. In this work, features related to fixations were derived from raw eye tracking data. Different machine learning classifiers were experimented to develop a better predictive model for dyslexia. From the experiments on different ML algorithms such as SVM, KNN and RF, it is observed that KNN achieved highest accuracy of $95 \%$ compared to other models.

Table- I: Classification results of different ML algorithms

\begin{tabular}{|c|c|c|c|c|c|c|}
\hline & \multicolumn{6}{|c|}{ Machine Learning Algorithms } \\
\hline & \multicolumn{4}{|c|}{ SVM } & \multirow[t]{2}{*}{ KNN } & \multirow[t]{2}{*}{ RF } \\
\hline & Linear & RBF & Sigmoid & Hybrid & & \\
\hline Accuracy & 0.93 & 0.93 & 0.92 & 0.93 & 0.94 & 0.91 \\
\hline Precision & 0.92 & 0.90 & 0.91 & 0.90 & 0.93 & 0.94 \\
\hline Recall & 0.94 & 0.97 & 0.94 & 0.96 & 0.96 & 0.88 \\
\hline Specificity & 0.92 & 0.87 & 0.89 & 0.88 & 0.92 & 0.94 \\
\hline F1 score & 0.93 & 0.93 & 0.92 & 0.93 & 0.95 & 0.90 \\
\hline
\end{tabular}

\section{REFERENCES}

1. C. Christo, J. M. Davis, and E.B. Stephen, Identifying, assessing, and treating dyslexia at school. Springer Science \& Business Media, 2009.

2. P. Ott, Teaching children with dyslexia: A practical guide. Routledge, 2007.

3. G. Reid, "Identification and assessment of dyslexia and planning for learning." in The Routledge companion to dyslexia, pp. 102-115. Routledge, 2012

4. B. Boets, H.P.O. de Beeck, M. Vandermosten, S. K. Scott, R. Celine Gillebert, D. Mantini, B. Jessica, S. Sunaert, W. Jan, and P. Ghesquière, "Intact but less accessible phonetic representations in adults with dyslexia." Science, vol. 342, pp. 1251-1254, 2013

5. S.E Shaywitz, Overcoming dyslexia: A new and complete science-based program for reading problems at any level. Knopf, 2003.

6. M. Iwabuchi, R. Hirabayashi, K. Nakamura \& N. K. Dim, Machine Learning Based Evaluation of Reading and Writing Difficulties. Studies in health technology and informatics, vol. 242, pp. 1001-1004, 2017

7. C. Billard and F. D. Pinton, "Clinique de la dyslexie. Archives de pédiatrie”, vol. 17, pp.1734-1743, 2010.

8. C. Prado, M. Dubois and S. Valdois, "The eye movements of dyslexic children during reading and visual search: impact of the visual attention span. Vision research", vol. 47, pp. 2521-2530, 2007.

9. J. Pan, M. Yan, J. Laubrock, H. Shu, \& R. Kliegl, "Saccade-target selection of dyslexic children when reading Chinese", Vision research, vol. 97, pp. 24-30, 2014.

10. B. Fischer, M. Biscaldi and P. Otto, "Saccadic eye movements of dyslexic adult subjects". Neuropsychologia, vol. 31, pp. 887-906, 1993

11. L. Rello and M. Ballesteros, "Detecting readers with dyslexia using machine learning with eye tracking measures" in Proceedings of the 12th Web for All Conference, ACM, pp. 16., May 2015.

12. J. Lustig, J. "Identifying dyslectic gaze pattern: Comparison of methods for identifying dyslectic readers based on eye movement patterns", 2016.

13. J. Bingel, M. Barrett \& S. Klerke, "Predicting misreadings from gaze in children with reading difficulties" in Proceedings of the Thirteenth Workshop on Innovative Use of NLP for Building Educational Applications , pp. 24-34, 2018

14. S. Kim \& R. Wiseheart, "Exploring Text and Icon Graph Interpretation in Students with Dyslexia: An Eye-tracking Study". Dyslexia, vol. 23, pp. 24-41, 2017. 
15. K. Lukasova, M. P. Nucci, R. M. N de Azevedo, G. Vieira, J.R. Sato \& E. Amaro, "Predictive saccades in children and adults: A combined fMRI and eye tracking study". PloS one, vol . 13, 2018.

16. H. Jarodzka and S. Brand- Gruwel, "Tracking the reading eye: towards a model of real-world reading.", Journal of Computer Assisted Learning, vol. 33, pp. 193-201, 2017.

17. J. Hautala, C. Kiili,, Y. Kammerer, O. Loberg, S. Hokkanen \& P.H Leppanen, "Sixth graders' evaluation strategies when reading Internet search results: an eye-tracking study". Behaviour \& Information Technology, pp. 1-13, 2018.

18. R. Kennedy and Eberhart, Particle swarm optimization, in Proceedings of IEEE International Conference on Neural Networks IV, vol. 1000, Nov 1995.

19. P. Charoen, W. Kongprawechnon \& K. Tungpimolrut, "A hybrid particle swarm optimization-SVM classification for automatic cardiac auscultation", Songklanakarin Journal of Science \& Technology, vol. 39, 2017.

20. Y. Mo \& S. Xu, Application of SVM based on hybrid kernel function in heart disease diagnoses in 2010 International Conference on Intelligent Computing and Cognitive Informatics, pp. 462-465, IEEE, June 2010

21. D. Lavanya \& D. K. U. Rani"Analysis of feature selection with classification: Breast cancer datasets." Indian Journal of Computer Science and Engineering, vol. 2, pp. 756-763, 2011.

22. D. A. Robinson, "The mechanics of human saccadic eye movement", The Journal of physiology, vol. 174, pp. 245-264, 1964

23. M. Vidal, J. Turner, A. Bulling \& H. Gellersen, "Wearable eye tracking for mental health monitoring." Computer Communications, vol. 35, pp. 1306-1311, 2012.

24. C. Galdi, M. Nappi, D. Riccio \& H. Wechsler, "Eye movement analysis for human authentication: a critical survey." Pattern Recognition Letters, vol. 84, pp. 272-283, 2016.

25. M. K. Eckstein, B. Guerra-Carrillo, M. Singley \& S. A Bung, "Beyond eye gaze: What else can eyetracking reveal about cognition and cognitive development?" Developmental cognitive neuroscience, vol. 25, pp. 69-91, 2017.

26. F. Y. Kuo, C. W. Hsu \& R. F. Day, "An exploratory study of cognitive effort involved in decision under Framing - an application of the eye-tracking technology.”, Decision Support Systems, vol. 48, pp. 81-91, 2009.

27. K. C. Fraser, K. L. Fors, D. Kokkinakis \& A. Nordlund, "An analysis of eye-movements during reading for the detection of mild cognitive impairment" in Proceedings of the 2017 Conference on Empirical Methods in Natural Language Processing, pp. 1016-1026, 2017.

28. M. Borys, M. Tokovarov, M. Wawrzyk, K. Wesołowska, M. W. Plechawska, R. Dmytruk \& M. Kaczorowska, "An analysis of eye-tracking and electroencephalography data for cognitive load measurement during arithmetic tasks", in Advanced Topics in Electrical Engineering 10th International Symposium , pp. 287-292, IEEE, March 2017.

29. U. Lahiri, Z. Warren \& N. Sarkar, "Design of a gaze-sensitive virtual social interactive system for children with autism", IEEE Transactions on Neural Systems and Rehabilitation Engineering, vol. 19, pp. 443-452, 2011

30. M. N. Benfatto, G. O. Seimyr, J. Ygge, T. Pansell, A. Rydberg \& C. Jacobson, C. Screening for dyslexia using eye tracking during reading. PloS one, vol. 11, 2016.

31. L. Kampermann, N. Wilming, A. Alink, C. Buchel \& S. Onat. "Aversive Learning Changes Face-Viewing Strategies", Model-Based Fixation -Pattern Similarity Analysis, 2017.

32. A. Al-Wabil, P. Zaphiris and S. Wilson, "Examining visual attention of dyslexics on web navigation structures with eye tracking". in International Conference on Innovations in Information Technology , Dec 2008

33. R. D. Elterman, L. A. Abel, R. B. Daroff, L.F. Dellosso \& J. L. Bornstein, J. L. Eye movement patterns in dyslexic children. Journal of learning disabilities, vol. 13, pp. 16-21.

34. A. Altaher, "Phishing websites classification using hybrid SVM and KNN approach", International Journal of Advanced Computer Science and Applications, vol. 8, 2017.

35. S. Iftikhar, K.. Fatima, A. Rehman, A. S. Almazyad \& T. Saba. "An evolution based hybrid approach for heart diseases classification and associated risk factors identification", Biomedical Research, vol. 28, pp. 3451-3455, 2017
36. C. H. H Yang, J. H. Huang, F. Liu, F. Y. Chiu, M. Gao, W. Lyu \& J. Tegner," A Novel Hybrid Machine Learning Model for Auto-Classification of Retinal Diseases" arXiv preprint arXiv:1806.06423, 2018.

37. T. Raiko, A. Ilin \& J. Karhunen "Principal component analysis for sparse high-dimensional data" in International Conference on Neural Information Processing, pp. 566-575 , Springer, Berlin, Heidelberg, Nov 2007

38. P. Luukka and J. Lampinen "A classification method based on principal component analysis and differential evolution algorithm applied for prediction diagnosis from clinical EMR heart data sets" in Computational Intelligence in Optimization , pp. 263-283, Springer, Berlin, Heidelberg, 2010.

39. Jolliffe, T. Ian and J. Cadima, "Principal component analysis: a review and recent developments." Philosophical Transactions of the Royal Society A: Mathematical, Physical and Engineering Sciences, vol. 374, pp. $2065,2016$.

40. A. Esmin, R. A. Coelho \& S. Matwin, "A review on particle swarm optimization algorithm and its variants to clustering high-dimensional data", Artificial Intelligence Review, vol. 44(1), pp. 23-45, 2015.

41. Jothi Prabha, A., \& Bhargavi, R. Prediction of Dyslexia from Eye Movements Using Machine Learning. IETE Journal of Research, 1-10, 2019

42. Jothi Prabha, A., \& Bhargavi, R., Ragala, R. Prediction of dyslexia using support vector machine in distributed environment. International Journal of Engineering \& Technology, 2795-2799, 2019

43. Prabha, A. J., \& Bhargavi, R. Prediction of Dyslexia Using Machine Learning-A Research Travelogue. In Proceedings of the Third International Conference on Microelectronics, Computing and Communication Systems , pp. 23-34. Springer, Singapore, 2019.

44. Frid A. \& Breznitz Z, "An SVM based algorithm for analysis and discrimination of dyslexic readers from regular readers using ERPs" in Electrical \& Electronics Engineers in Israel,IEEE 27th Convention of pp. 1-4, 2019.

45. Guyon, I., Weston, J., Barnhill, S., \& Vapnik, V. Gene selection for cancer classification using support vector machines. Machine learning, 46(1-3), 389-422, 2002.

46. Huang, M. L., Hung, Y. H., Lee, W. M., Li, R. K., \& Jiang, B. R. (2014). SVM-RFE based feature selection and Taguchi parameters optimization for multiclass SVM classifier. The Scientific World Journal, 2014

\section{AUTHORS PROFILE}

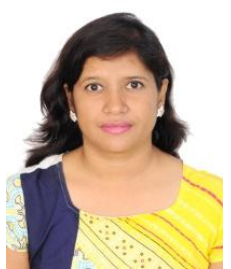

Jothi Prabha. A (Jothi Prabha Appadurai) obtained her bachelor's degree in Information Technology from Bharathidasan University in the year 2002 and master's degree in Software Engineering from Jawaharlal Nehru Technological University in the year 2012. She has 6 years of Industry experience as a Software Engineer at Bank of America Continuum solutions, Hyderabad and 9 years of Academic experience as Associate Professor in CSE Department at Jyothishmathi Institute of Technology and Science. Currently, she is a full time Research scholar at School of Computing Science and Engineering, VIT University, Chennai Campus, India. Her current research interests are Data Mining and Data Analytics.

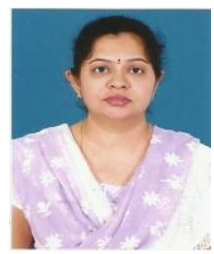

Bhargavi R (Bhargavi Rentachintala) is presently working as Associate Professor in the School of Computing Science and Engineering, VIT University, Chennai Campus, India. She has more than 20 years of Industry, Academic and Research experience She received her M.Tech and $\mathrm{Ph} . \mathrm{D}$ degrees from IIT Madras and Anna University respectively. Her research interests include Complex Event Processing, Machine learning, in Healthcare and Data Science. She has authored and published several research papers in IEEE/ACM/Springer international conferences and refereed Journals. She also authored chapters in highly reputed research reference books

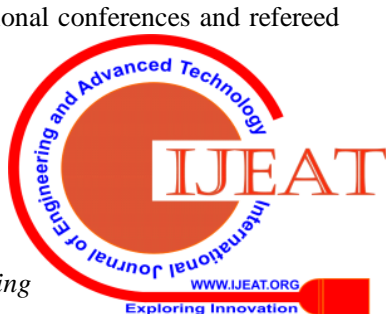


Predictive Model for Dyslexia from Eye Fixation Events

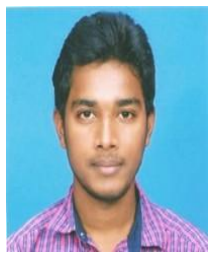

Harish B is currently pursuing his Master's (Integrated) in Software Engineering in the School of Computing Science and Engineering, VIT University, Chennai Campus, India. He is currently working in the development of Machine Learning Library for Open Matrix Language. His research interests include Machine Learning and Deep Learning. 